\title{
Modelo de Educación Histórica y formación docente
}

\author{
Model of historical education and teacher training \\ Modelo de educação histórica e formação docente
}

Gerardo Daniel Mora Hernández ${ }^{1}$

Rosa Ortiz Paz ${ }^{2}$

\section{RESUMEN}

En México el Modelo Educación Histórica es una propuesta didáctica que se basa en la interacción con fuentes primarias y el uso de conceptos analíticos ("de segundo orden"). Como modelo formativo parte de las "representaciones" de los estudiantes normalistas de la historia y su enseñanza -tradicionalistasconfrontadas con actividades vivenciales. Sigue con el aprendizaje procedimental del modelo didáctico. Y concluye con el Trabajo Docente en condiciones reales ("aprendizaje situado"), la redacción de un "documento recepcional" y el examen profesional (docencia reflexiva). Este modelo formativo lo hemos desarrollado en la Especial idad de Historia de la Licenciatura de Educación Secundaria en la Escuela Normal Superior de México desde 2006, asesorando a un centenar de estudiantes.

Palavras-chave: Modelo de educación histórica. Representaciones. Modelo formativo.

\section{ABSTRACT}

In Mexico Model Historical Education is an educational proposal that is based on the interaction with primary sources and use of analytical concepts ("second order"). Training model as part of the "representations" of student teachers of history and teaching -tradicionalistas- confronted with experiential activities. Follow the procedural learning tea ching model. He concludes with the teaching work in real conditions ("situated learning"), the drafting of a "receptional document" and the professional examination (reflexive teaching).This training model we have developed in the specialty of History of Bachelor of Secondary Education in Higher Normal School of Mexico since 2006, advising a hundred students.

Keyword: Model of historical education. Representations. Training model.

\footnotetext{
${ }^{1}$ Escuela Normal Superior de México. Cuerpo Académico de Educación Histórica.

${ }^{2}$ Uni ve rsidad Autónima Metropolitana IztapalapA. Escuela Normal Superior de Mé xico. Cuerpo Académico de Educación Histórica.
} 


\section{RESUMO}

No México, Modelo de Educação histórica é uma proposta educacional que se baseia na interação com fontes primárias e o uso de conceitos analíticos (conceitos de "segunda ordem"). Modelo de formação parte das "representações" de professores estudantes de história e ensino -tradicionalistas-confrontado com atividades vivenciais. Segue o modelo de ensino aprendizagem processual. Cosnidera o trabalho de ensino em condições reais ("aprendizagem situada"), a elaboração de um "documento sistematizador" e o exame profissional (ensino reflexivo). Este modelo de formação, temos desenvolvido na especialidade de História no Bacharelado em Educação Secundária na Escola Normal Superior do México desde 2006, aconselhando uma centena de alunos.

Keyword: Modelo de educação histórica. Representações. Modelo de formação..

\section{La crisis de la formación docente en Historia.}

Formar a profesores de Historia para la educación secundaria es un proceso complejo que requiere determinados perfiles de ingreso, un currículo consistente, profesores, materiales, gestión curricular y óptimas condiciones para las prácticas docentes, elementos que nunca se conjuntan.

Si bien es cierto que el Plan de la Especialidad de Historia de la Licenciatura en Educación Secundaria de 1999 es mejor que sus precedentes por incluir la práctica docente en la mayoría de los semestres, la escasez de capacitación a los formadores, materiales específicos y actualización de los programas, limitaron sus resultados. Especialmente porque prevaleció al menos en la Normal Superior del DF- la separación entre las contenidos de las materias de Historia y las de práctica docente, incluyendo a las "didácticas" - denominadas "Historia y su enseñanza" pretendiendo darles un nuevo enfoque. En las primeras prevalece una historia narrativa, que debe reproducir el estudiante para "dar clase", usando manuales, fotocopias y material de la web. En las segundas se confiaba en la formación artesanal (observación y práctica supervisada por profesores de secundaria y normalistas).

El Plan comenzó en 1999, cuando la formación artesanal estaba superada por otros paradigmas como los prácticos reflexivos y la didáctica de la Historia ya había superado el paradigma transmisivo. Recordemos que este Plan 1999 nunca fue actualizado, aunque los de las licenciaturas en preescolar y primaria ya se renovaron.

Las deficiencias del currículo formal y las carencias de su aplicación tuvieron como resultado profesores con insuficientes conocimientos disciplinarios y pedagógicos que les permitieran reflexionar y mejorar su práctica docente. Las notables excepciones eran 
producto del diseño y realización de un trayecto formativo paralelo al currículo formal, porque las escuelas normales legalmente no lo pueden modificar.

Respecto a la formación continua, por diversas razones hay un escaso impacto de los diversos cursos, diplomados y estudios de posgrado en la enseñanza de la Historia. Tal vez por su academicismo o por el desconocimiento de los nuevos problemas de la práctica docente. En consecuencia, la formación docente en Historia se encuentra en crisis, incluyendo a los paradigmas formativos que la han orientado hasta el momento: artesanal y mixto (estudios separados de historia y pedagogía).

\section{El imaginario de la cátedra}

Si consideramos que la mayoría de los estudiantes normalistas sólo tienen estudios de bachillerato, y que han recibido una enseñanza transmisiva de la Historia, su representación común de la buena enseñanza es la clase expositiva, utilizando los recursos que requiera la narración. Las habilidades retóricas e histriónicas resultan imprescindibles. Esta imagen se refuerza en la escuela normal en las clases escolarizadas, de ahí la tendencia del estudiante a "exponer" en su práctica docente.

El imaginario de los estudiantes al ingresar a la Especialidad de Historia es que para ser profesor se necesita "saber historia" (narrativas) y luego exponer sus conocimientos ante un grupo utilizando técnicas y recursos didácticos. Esta representación la comparten los formadores y la reproducen con los estudiantes. Tratamos de formar discípulos que sigan nuestro estilo docente y, particularmente, nuestra ideología, causando el efecto Pigmalión.

Estas creencias son muy difíciles de modificar. Son similares al sentido común con que se intenta comprender al tiempo histórico, por lo que se requieren estrategias contraintuitivas para superarlas. Estas "representaciones" -que se convierten en rutinasrequieren revertirse con métodos activos e innovadores (LICERAS RUIZ, 2004).

Cabe mencionar que esta imagen del "catedrático" (especialista en alguna temática de Historia) al aplicarse en la escuela secundaria hace estragos en la autoestima docente, llevándole a crisis de identidad profesional y a la pérdida de sus ideales vocacionales, incluso a su huida de la docencia (con becas-comisión, puestos directivos o sindicales o emigrando a otros niveles educativos). 


\section{El retorno de la Especialidad de Historia a la escuela normal.}

Con el Plan 1983 -que transformó los estudios normalistas en licenciaturas- se suprimió la Especialidad e Historia y se integró el área de Ciencias Sociales con Historia, Geografía y Civismo.

En 1993 la Historia retornó al plan de educación secundaria con tres cursos. En la Escuela Normal Superior de México realizamos "un programa emergente" porque fue hasta el ciclo 1999-2000 que se presentó el currículo para tales Especialidades. Pero con el cambio de los planes y la renovación de la plantilla docente, se perdieron la "cátedra", las tesis de temas históricos y varias materias de Historia.

La didáctica que se impartía en las escuelas normales trataba de superar los problemas de la enseñanza expositiva de una Historia enciclopédica. Vale recordar las sugerencias que se hicieron en el plan y programas de las escuelas secundarias de 1964:

Evitar las largas disertaciones. Evitar los dictados hasta donde las condiciones lo permitan.

Provocar la intervención activa del alumno, mediante: Elaboración de monografías: hombres famosos, etapas históricas, países, etc. Lecturas, dramatizaciones, etc. Visitas a museos y lugares históricos. Intervención en la conmemoración de fechas y hechos históricos. Colección de objetos históricos. Formación de álbumes con recortes de periódicos, fotografías, etc. Concursos históricos literarios. Formación de sencillas exposiciones periódicas.

Utilización constante de material objetivo y audiovisual. Cartografía adecuada, elaboración de mapas y esquemas. Colección de recortes de información y grabados periodísticos. Utilización del cine de placas fijas y móviles, sonoro y mudo. Discos grabados.

Aprovechar las posibles conexiones de la enseñanza histórica con las demás actividades escolares de los alumnos. 
Selección de los hechos históricos de acuerdo a su trascendencia en relación con el progreso de la humanidad. (MORA; ORTIZ PAZ, 2012a).

Pero en el programa 2006 de la asignatura, casi medio siglo después de los consejos precedentes y ya con el enfoque por competencias, se recomendaba al docente: "Enfatizar el análisis y la comprensión histórica. No debe abusarse de la exposición, el dictado, la copia fiel de textos y la memorización pasiva."

Estas prácticas rutinarias continúan y causan apatía y rechazo hacia la Historia. En estas condiciones las prácticas docentes (jornadas de 8 a 12 clases) son difíciles, sobre todo en los últimos dos semestres cuando se realiza el Trabajo Docente en condiciones reales. Los practicantes buscan estrategias y actividades de cualesquier tipo (juegos, computación, cine, documentales, historietas, escenificaciones, mapas conceptuales, etc.) para mantener el interés de sus alumnos. Esto se comprueba con los documentos recepcionales (informes de propuestas didácticas aplicadas).

Esta continuidad de la didáctica tradicional no fue menguada por el enfoque constructivista que se estableció en las cinco materias de "Historia y su enseñanza". La causa principal de ello fue la desvinculación entre las nueve "Historias" (2 universales, 3 de México, 2 seminarios y 2 optativas) de contenido narrativo, las de práctica docente expositiva (divididas en 2 de Observación, 4 de Prácticas y 2 de Trabajo Docente) y las 5 didácticas. Asimismo porque el enfoque constructivista no logró permear en los libros de texto y los demás "recursos didácticos", ni en la actualización docente. Un buen ejemplo es que en Tercer semestre se inician las prácticas con Historia I (mundial) y esta materia se imparte en Cuarto semestre, como Planeación y evaluación. Aunque también debe destacarse que la bibliografía propuesta de "Historia y su enseñanza" no lograba sistematizar un modelo didáctico alternativo a la clase magistral. Como sostiene Santisteban (2007, p. 25):

Existe una problemática generalizada en la realización de las secuencias didácticas. Los estudiantes de maestro tienen dificultades para estructurar sus propuestas para la enseñanza del tiempo histórico, ya que no disponen de un modelo inicial de referencia. Por este motivo tienden a centrarse en conocimientos factuales del estudio de la historia que ya dominan, para estructurar a su alrededor los contenidos. Se alejan así de un modelo conceptual de enseñanza y aprendizaje de la historia. 
En consecuencia, el practicante durante el Trabajo Docente realiza exposiciones apoyadas en algunos recursos (ilustraciones fotocopiadas, mapas, presentaciones y audiovisuales cuando se cuenta con el equipo).

En síntesis, las limitaciones del currículo 1999 de la Especialidad de Historia - un tronco común desproporcionado, insuficiente número de prácticas docentes a pesar de sustentarse en el paradigma formativo "artesanal", desvinculación de las materias de la Especialidad, entre otras- no han permitido una renovación de las prácticas. Las causas son similares a las de un caso español (LICERAS RUIZ, 2004, p. 12):

a) la concepción que tienen los alumnos de la profesión de enseñar, basada fundamentalmente en el modelo tradicional;

b) las pocas posibilidades de participar y actuar en experiencias educativas diferentes de ese modelo;

c) el poco tiempo para la reflexión y la relación de la teoría con la práctica;

d) la perdurabilidad de las ideas previas, a pesar del proceso de formación, dificulta la capacidad de reflexionar sobre la propia práctica;

e) el escaso desarrollo de una actitud profesional, que podría facilitarla construcción de una actitud de revisión, de análisis y de actuación constante para mejorar permanentemente la labor docente; $y$

f) la necesidad de plantear unos planes de formación de profesores integrales y coherentes que permitan establecer la reflexión como hábito profesional y método de toma de decisiones.

\section{Hacia un nuevo paradigma formativo.}

Encontramos tres paradigmas educativos de la Historia:

- analíticos e investigativos, como el aprendizaje basado en problemas y los "conceptos de segundo orden"; 
- socioculturales, como la educación patrimonial y el aprendizaje basado en proyectos;

- piscopedagógicos, como el "constructivismo" y el cambio conceptual.

Cada paradigma requiere un modelo formativo. No obstante, conviven diversos modelos que involucran los estudios de Historia, paralelos o previos a la formación pedagógica, continuada con periodos de práctica docente. Y sólo en el posgrado se realiza investigación y, en menos casos, innovación. También hay actividades formativas en museos, asociaciones profesionales y grupos académicos.

Guyver y Nichol (2004, p. 3) específica los conocimientos necesarios en la formación inicial:

\author{
Temas históricos; \\ Métodos de investigación y análisis históricos (syntactic subject);; \\ Creencias (representaciones) sobre la Historia; \\ Diseño Curricular; \\ Pedagógico; \\ Modelos de enseñanza ; \\ Procesos cognitivos de los alumnos; \\ Comportamiento escolar de los alumnos \\ Reflexión (knowledge of self); \\ Contextos escolares; \\ Propósitos educativos (educational ends).
}

Pagès (2000, p.157) señala los fines de la formación inicial:

En mi opinión, preparar a un profesor o una profesora de historia, enseñar a enseñar historia, consiste en educar a un profesional para que tome decisiones, sepa organizarlas y llevarlas a la práctica, sobre los conocimientos históricos que debe enseñar en un aula de un centro 
determinado. Aprenda que enseñar historia en secundaria consiste en preparar a los jóvenes para que se sitúen en su mundo, sepan interpretarlo desde su historicidad y quieran intervenir en él con conocimiento de causa, quieran ser protagonistas del devenir histórico.

No obstante, el problema es cómo lograr estos fines, considerando que las "representaciones" y rutinas de los estudiantes normalistas los limitan, por decir lo menos. Dalongeville (2003, p. 5) precisa que el profesor debe:

a) prever una situación para que puedan emerger las representaciones del sujeto;

b) analizar las representaciones, un trabajo para el que raramente los profesores están formados;

c) elaborar una situación (fases individuales, semicolectivas y colectivas) que le va a resultar dificultosa al sujeto-discente y hacer de manera que las representaciones que tenga no le sean suficientes para ir más allá del punto muerto al que le han conducido sus representaciones iniciales;

d) movilizar materiales (documentos de todo tipo, consignas de trabajo) que fomenten lo que denomino disonancia cognitiva.

En este sentido, la formación también debe ser contraintuitiva: a partir del cuestionamiento de sus representaciones con la interacción sensible y racional con las fuentes primarias; la investigación en la escuela; la práctica "simulada" y "situada" con un modelo educativo que confronte sus rutinas expositivas, y en comunidades de aprendizaje que fomenten su reflexión con la literatura especializada. Esta propuesta es factible con un currículo modular más vinculado a las escuelas básicas (más allá de las dos semanas de práctica en Observación y Práctica Docente I-IV), a la investigación histórica y pedagógica, y a los medios de difusión de la Historia.

En consecuencia, las materias de Historia deben enfocarse más a los problemas, los métodos y las fuentes primarias que a la narrativa de los manuales escolares - que transcriben otras interpretaciones. Y las materias de práctica docente integrarse con contenidos teóricos (didácticos), de investigación educativa (el aprendizaje de Historia en las aulas) y diseño, planificación, realización y evaluación de secuencias de enseñanza. Ambas en módulos progresivos que permitan primero el conocimiento de la realidad escolar, luego las prácticas para desarrollar habilidades docentes, y finalmente el Trabajo Docente enfocado a la resolución de un problema de la práctica o a la innovación. 


\section{El modelo formativo de Educación Histórica.}

Como asesores de Trabajo Docente, en principio recuperamos propuestas que ya se habían formalizado en las Didácticas de Historia: visitas a museos, cine, historietas, dramatizaciones, juegos. Todas estas resultaban eficaces al momento, pero al persistir la transmisión en la clase se volvía al aburrimiento y a las medidas de "control" de grupo. Por lo que era necesario integrar estas propuestas a lo la rgo del año escolar y modificar la rutina de la clase. El cambio de programas en 2006 nos permitió hacer una primera propuesta que fuimos desarrollando hasta el año escolar 2011-2012.

El uso de fuentes en las clases era una necesidad insoslayable, ya que los alumnos cuentan con una cultura audiovisual con la que la sola narrativa docente no puede competir. La clase debía ser siempre interactiva con estas fuentes, tratando de simular el entorno virtual que le proporciona la tecnología de la que es nativo (televisión, videojuegos, computadora, celulares, etc.).

Si bien es cierto que ya se habían usado los mapas conceptuales y otros organizadores gráficos en la enseñanza de la Historia, introdujimos en la clase a las "representaciones no lingüísticas" propuestas por Marzano (2000). Con nuevos organizadores y una técnica de enseñanza que los convertía en "estrategias de aprendizaje", conformamos la base de nuestro modelo didáctico: la "clase interactiva". Su aplicación sustituye al dictado, permite ordenar la información del tema y su exposición, volviéndose comprensible al alumno. Cabe mencionar que han tenido éxito no sólo con alumnos avanzados, sino con alumnos con deficiencias de lectura y escritura. ¿Es posible esto? Sí, porque la cultura de los alumnos es audiovisual y virtual.

En 2011 con el cambio curricular de la asignatura de Historia en educación secundaria, incluimos en nuestro modelo los conceptos analíticos, denominados de segundo orden. El modelo es un conjunto de principios (la interacción con las fuentes primarias), estrategias (esquemática y talleres cooperativos, la didáctica multimedia) e instrumentos de diagnóstico grupal (matriz de aprendizaje) para diseñar secuencias didácticas que se aplican progresivamente durante todo el año escolar, con la finalidad de formar competencias históricas "situadas" (DÍAZ-BARRIGA, 2006) de acuerdo a cada contexto escolar.

Consideramos que es una innovación porque supera la enseñanza transmisiva de "historias" (narrativas) y lo hemos fundamentado respecto al qué enseñar, a quién, cómo, cuándo y por qué (MORA; ORTIZ PAZ, 2012a, 2012b, 2013)³. Tenemos diversas evidencias de su

\footnotetext{
${ }^{3}$ https://sepdf.academia.edu/GerardoMora
} 
aplicación como documentos recepcionales, videograbaciones de prácticas, fotografías de cuadernos de los alumnos de secundaria, y portafolios de estudiantes normalistas.

Como modelo formativo, se concentra en el desarrollo de tres habilidades docentes: "la transposición didáctica" o dominio de la historiografía para el diseño de una secuencia didáctica; "la enseñanza estratégica" o capacidad de realizar dicha secuencia superando los problemas emergentes de la clase; y la "docencia reflexiva" o capacidad de autoevaluación y mejora.

Para Observación y Práctica Docente I-IV - que se imparten del segundo al sexto semestre- y el Taller de diseño de propuestas didácticas y análisis del trabajo docente I-II que se imparte en el último año de la Especialidad- hemos diseñado instrumentos de evaluación, formatos de planificación, guías didácticas y textos explicativos. Con ellos el practicante realiza el diagnóstico de su grupo, para definir las estrategias de gestión del ambiente (reglas, negociación, actividades y autonomía con retos), de contenidos (niveles de dominio básico, intermedio o avanzado de los aprendizajes esperados) y didácticas (actividades de interacción sensible y racional). Posteriormente diseña una secuencia con inicio, desarrollo y cierre evaluativo. Prepara la planificación y materiales. Coordina la secuencia, resolviendo los problemas emergentes de la clase. Y evalúa los resultados, tanto de los aprendizajes esperados como de la enseñanza. Videograbamos algunas y las evaluamos en grupo, utilizando una rúbrica. Por último, coevaluamos el informe de la práctica, incluyendo sus propias propuestas de mejora.

Con este modelo orientamos el Trabajo Docente. No "calificamos" resultados de la práctica, sino la interiorización del proceso reflexivo en cada estudiante. Nuestra función es de asesoramiento y a compañamiento ante los problemas de la práctica. Conformamos así una comunidad de aprendizaje que comparte éxitos y fracasos, convirtiéndoles en experiencia docente.

Respecto a las materias de Historia y las de su enseñanza, privilegiamos el contacto con fuentes primarias, visitando museos y sitios históricos. Realizando diversas actividades y prácticas simuladas (guías o clases realizadas por los estudiantes). Así también con el uso de cine y documentales que ayuden a la contextualización de los temas. Y fichas que ayuden a los alumnos a vincular los contenidos históricos (conceptos sustantivos y metodológicos) con las fuentes y las actividades didácticas con las que podrá diseñar una secuencia.

Por supuesto, este ha sido un proceso con altibajos. Existen grandes dificultades en cambiar las representaciones y rutinas expositivas de los estudiantes, conflictos con otros profesores por diferencias pedagógicas e ideológicas, escasez de recursos para las visitas y prácticas, libros, equipos tecnológicos, etc. Cabe destacar la discusión sobre los contenidos históricos a enseñar, tanto en la escuela normal como en la escuela secundaria. 
La llamada "historia de bronce" exalta a los héroes nacionales, la "crítica" denuncia las injusticias, y la "científica" pretende la objetividad mediante la comprobación. En la secundaria prevalece la historia de bronce, en tanto que en Normal la historia crítica, no teniendo acuerdo sobre cuál de sus versiones. Las divergencias son entre una propagandística, cuasi doctrinaria, y otra reflexiva que recurra a las evidencias y la argumentación.

Tal vez la reflexión más importante es que el cambio de representaciones que tienen los estudiantes no es un proceso lineal o progresivo, que se logre sólo con aprobar cursos, talleres y seminarios. En algunos normalistas se logra con las actividades de sensibilización y con la aplicación del modelo de educación histórica en la escuela secundaria. Pero éstos no siempre logran su dominio integral, enfocándose unos a los procedimientos y otros a los talleres, ni el proceso reflexivo.

Estos resultados nos llevan a comprender la trayectoria de los estudiantes normalistas en función de varios factores, empezando con sus intereses profesionales y personales, así como las condiciones económicas y familiares que condicionan su desempeño. En segundo lugar, por sus conocimientos. En un estudio reciente, se demuestra que son muy limitados, incluso ya en semestres avanzados. Por otra parte, la atomización de los formadores y el rezago en su actualización son un factor determinante en el perfil de egreso. En efecto, los estudiantes más formados son casos particulares.

\section{Un caso exitoso de formación.}

Martín Palomino Curiel realizó sus prácticas profesionales en la Escuela Secundaria Diurna $\mathrm{N}^{\circ} 38$ del Distrito Federal, bajo la tutoría del Prof. Juan Carlos Olivares Orozco, un profesor experto con 28 años de experiencia docente, contando además con un salón de Historia donde se realizan las clases. Así la dinámica grupal fomenta la autonomía del aprendizaje, a pesar de que la mayoría de alumnos sean pasivos.

El orden del diseño de las secuencias didácticas que hizo fue el siguiente:

- diagnóstico del grupo de acuerdo a la matriz de aprendizaje escolar;

- transformar el Aprendizaje Esperado de cada tema en un concepto de segundo orden; 
- definir el nivel de dominio procedimental adecuado al grupo (conocimiento, práctica o aplicación);

- elaborar la pregunta problematizadora;

- diseñar la actividad de apertura (sensibilización con fuentes primarias);

- seleccionar el esquema u organizador gráfico con sus palabras clave del tema;

- y especificar los criterios de evaluación según el nivel de dominio procedimental.

Estas secuencias conforman la "clase interactiva" (actividad de sensibilización, análisis de información con esquemas y cierre evaluativo del aprendizaje esperado) y las "actividades de integración" (talleres y proyectos autónomos de inteligencias múltiples, museología, periodismo y dramatización). Su planificación combina actividades y clases.

Esta adaptación del modelo educativo a los conceptos de segundo orden permitió que el practicante desarrollara sus habilidades docentes evitando la rutinización y el espontaneísmo. Pero lo importante de este caso es que vinculó nuestra propuesta didáctica, con la experiencia de su tutor y el nuevo enfoque de los programas 2011, logrando una innovación. En particular, descubrió algunos principios del "aprendizaje multimedia" (RIVERO GRACIA, 2011), para articular en la enseñanza los lenguajes oral, escrito y visual. Todo ello se puede encontrar en su documento recepcional y su examen4, así como en videos de sus prácticas.

Este proceso implicó superar un conjunto de dificultades:

- La enseñanza expositiva.

- El conocimiento de los alumnos para lograr su adecuada clasificación.

- La enseñanza de los esquemas como procedimientos.

- El conocimiento de fuentes primarias para cada tema.

- El diseño de secuencias interactivas.

- La habilidad para elaborar materiales.

\footnotetext{
${ }^{4}$ https://www.youtube.com/user/gdmhmx/videos
} 
- La organización del grupo escolar.

- Evaluar los aprendizajes esperados.

- Reflexionar sobre la práctica docente.

\section{Conclusiones}

1. Cada didáctica de la Historia implica un modelo formativo de sus profesores de nivel básico, expresándose en currículos escolarizados y la actualización docente. En México es posible observar la crisis de los modelos "artesanal" (observación y práctica docente) que opera en las escuelas normales, y "mixto" (estudios generales de Historia y cursos de didáctica), trayecto seguido por los profesores que no son normalistas. El modelo artesanal no supera las rutinas expositivas hegemónicas en las aulas y crea un déficit de conocimientos disciplinarios, en tanto que el mixto lo crea de conocimientos pedagógicos. Se requiere de un nuevo paradigma formativo.

2. Aunque existe coincidencia en los conocimientos y el perfil que un profesor de Historia debe tener, el problema de cómo lograrlos aún no se ha resuelto. Además de las limitaciones extraescolares, del currículo, de los conocimientos previos de los estudiantes y de los formadores, las "representaciones" sobre la docencia (la cátedra magistral)y las rutinas transmisivas de enseñanza permean la operación de los currículos normalistas.

3. Acordes con los notables avances de la disciplina y la investigación educativa, un nuevo modelo formativo puede delinearse integrando los resultados de las nuevas metodologías de la Historia y la didácticas deconstructivas de las "representaciones" escolares del pasado.

4. La formación inicial requiere la interacción sensible y analítica con las fuentes primarias - principio esencial de la Educación Histórica- para realizar la "transposición didáctica", superando la memorización de contenidos positivistas ("hechos") y relatos cronológicos.

5. El conocimiento del contexto escolar y de los alumnos de básica necesita trascender las metodología pasivas, sólo observar y describir sin evaluar, 
incorporando los resultados sobre progresión del pensamiento histórico (Lee, Shemilt) y las didácticas deconstructivas (Dalongeville, Winburg, VanSledright).

6. Es indispensable un modelo didáctico base para el aprendizaje procedimental (conocimiento, práctica y aplicación) de habilidades docentes. Éstas se logran mediante prácticas simuladas (en el aula normalista) y situadas (en la escuela secundaria), debiendo evaluarse de manera reflexiva para su mejora. En términos del currículo vigente para la Especialidad de Historia, se requiere incluirlo en las materias didácticas ("Enseñanza de la Historia") y operarlo en las de práctica docente. Y en un nuevo currículo, acorde con los nuevos programas de básica, integrar ambos contenidos en una sola materia.

7. El Modelo de Educación Histórica ha permitido el desarrollo de habilidades docentes (planificación, evaluación y gestión didáctica) de un centenar de estudiantes normalistas en el contexto de las secundarias públicas del Distrito Federal. Sólo en casos especiales se logra la reflexión docente, debido a las representaciones tradicionales de la Historia y su enseñanza.

\section{Referencias}

DALONGEVILLE, Alain. Noción y práctica de la situación problema en Historia.Enseñanza de las ciencias sociales, Barcelona, n .2, p. 3-12, 2003.

DÍAZ-BARRIGA, Frida.Enseñanza situada: Vínculo entre la escuela y la vida. México. McGrawHill, 2006.

GUYVER, Robert; NICHOL, Jon. From novice to effective teacher: a study of postgraduate training and History pedagogy. International Journal of Historical Learning, Teaching and Research, New York, v. 4, n. 1, 2004.

LICERAS RUIZ, Ángel. La investigación sobre formación del profesorado en didáctica de las ciencias sociales. Profesorado, revista de currículum y formación del profesorados, Granada, v. 8 n. 1, 2004.

Marzano, Robert. What works in classroom instruction. USA: Mc Rel, 2000.

MORA, Gerardo; ORTIZ PAZ, Rosa. O modelo da Educação Histórica: experiencia de inovação para a educação básica. História \& Ensino, Londrina, v. 18, n. 1, p. 219-237, jan./jun. 2012a. 
. El Modelo de Educación Histórica. Experiencia de innovación para educación básica en México. Enseñanza de las ciencias Sociales, Barcelona, n.11, p. 87-98, 2012b.

_ _ _ _ _ _. La enseñanza del "tiempo histórico": problemas y propuestas didácticas. História \& Ensino, Londrina, v. 19, n. 1, p. 29-47, jan./jun., 2013.

PAGÈS, Joan. La formación inicial del profesorado para la enseñanza del patrimonio histórico y de la historia. Treballs d'Arqueologia, Barcelona, n. 6, p. 205-217, 2000.

Enseñar a enseñar historia: la formación didáctica de los futuros profesores de historia. In: GÓMEZ-HERNÁNDEZ, José-Antonio; MARÍN, María Encarna Nicolás. (Coord.). Miradas a la Historia. Murcia: Universidad de Murcia, 2004. p. 155-178. Aula de debate.

RIVERO GRACIA, Maria Pilar. El aprendizaje multimedia de la historia: valoración de una experiencia de aula. In: LÓPEZ FACAL, R.; VELASCO, L. (Ed.). Pensar históricamente en tiempos de globalización. Investigaciones sobre enseñanza y aprendizaje de la historia y las ciencias sociales. España: Universidad de Santiago de Compostela, 2011.

SANTIESTEBAN, Antoni. Una investigación sobre cómo se aprende a enseñar el tiempo histórico. Enseñanza de las ciencias sociales, Barcelona, n. 6, p. 19-29, 2007.

Recebido em 06.07.2016

Aprovado em 15.09.2016 